\title{
O modelo de Solow-Swan na linguagem de dinâmica de sistemas: uma aplicação para o Brasil
}

\author{
Newton Paulo Bueno \\ Departamento de Economia \\ da Universidade Federal de Viçosa
}

\section{Palauras-chave}

dinâmica de sistemas, macroeconomia, modelo Solow-Swan, Brasil.

\section{Classificação JEL O41.}

Key words

system dynamics, macroeconomics, Solow-Swan model, Brazil.

JEL Classification $O 41$.

\section{Resumo}

O objetivo deste texto é contribuir para a discussão sobre o crescimento econômico brasileiro utilizando uma abordagem inédita: a dinâmica de sistemas. Especificamente, pretende-se desenvolver uma versão sistêmica do modelo de Solow-Swan que permita explorar novos ângulos de insights de trabalhos que têm empregado o modelo para o Brasil e avaliar as possibilidades de crescimento da economia brasileira no futuro próximo. A motivação para abordar o tema com essa metodologia é que ela permite modelar o crescimento econômico como um processo dinamicamente complexo. Mostrar-se-á que abordar o tema dessa perspectiva abre uma gama de novas possibilidades no que diz respeito à experimentação com modelos teóricos, tais como proceder a análises mais acuradas de sensibilidade de soluções e obter estimativas para parâmetros não observáveis, por meio do procedimento de calibragem.

\section{Abstract}

This paper aims at contributing to the Brazilian economic growth discussion based on a new approach: system dynamics. Specifically, we intend to develop a systemic version of Solow-Swan model which allows the exploration of new angles of some important insights from recent Brazilian studies using that model and the assessment of the ability of the economy to grow in the near future. The reason for approaching the problem from that perspective is that it allows modeling economic growth as a complex dynamic process. We shall argue that studying the issue from the systemic perspective may open new avenues of research such as allowing the performance of more accurate sensitivity analysis and the obtention of more reliable forecasts for non observable parameters by means of calibration procedure. 


\section{1_Introdução}

Após um período em que pareceu que os métodos de previsão não estruturais por si sós seriam capazes de permitir aos economistas inferir os movimentos das variáveis macroeconômicas diretamente das séries de dados com um mínimo de teoria, é possível detectar uma revalorização dos modelos teóricos em gerar previsões passíveis de ser confrontadas com a evidência empírica. Diferentemente dos antigos modelos estruturais da década de 1960, entretanto, os modelos atuais tendem a ser de menor escala e mais fundamentados microeconomicamente (Diebold, 1998).

Assim é que, tanto na vertente novo-clássica, empregando modelos dinâmicos de equilíbrio geral, como os de ciclo real de Kydland e Prescott (1982 e 1996), ou modelos de crescimento endógeno (Kremer, 1993), como na vertente neokeynesiana, modelos de dimensão relativamente reduzida e estruturalmente simples têm sido utilizados para estudar o processo de crescimento econômico. Nesta segunda vertente, especificamente, textos importantes têm chegado à conclusão um tanto inesperada de que o modelo de Solow-Swan gera previsões em geral consistentes com a evidência empírica para períodos mais longos.
Barro e Sala-i-Martin (2004, cap.12), por exemplo, concluem que, para uma ampla amostra de países, é possível detectar uma tendência de convergência condicional das taxas de crescimento do PIB per capita. Em uma perspectiva Brasileira, Bacha e Bonelli (2005) prevêem que a taxa de crescimento equilibrado derivada do modelo Solow-Swan estabelece um limite para a acumulação de capital para o Brasil, dado pela taxa de aproximadamente 4\%, o qual somente poderia ser superado por uma aceleração da taxa de progresso técnico.

O objetivo deste texto é contribuir para essa discussão utilizando uma abordagem ainda não empregada pelos economistas brasileiros: a dinâmica de sistemas. Especificamente, pretende-se desenvolver uma versão sistêmica do modelo de Solow-Swan para avaliar as possibilidades de crescimento da economia brasileira no futuro próximo, bem como para identificar eventuais obstáculos e pontos de alavancagem atuais para o crescimento.

A motivação para abordar o tema com essa metodologia é que ela permite modelar o crescimento econômico como um processo dinamicamente complexo, em que o fato de as variáveis macroeconômicas encontrarem-se interligadas 
com defasagens temporais em ciclos de retroalimentação pode produzir resultados contraintuitivos, não identificáveis $a$ priori com base em uma abordagem não sistêmica. Mesmo quando esses resultados são consistentes com os obtidos com outras metodologias, como se verá que será o caso deste trabalho, abordar o tema dessa perspectiva abre uma gama de novas possibilidades no que diz respeito à experimentação com modelos teóricos. Por exemplo, a metodologia de dinâmica de sistemas é uma ferramenta altamente flexível para modelar relações não lineares e simular o comportamento dos sistemas correspondentes sob diferentes especificações dessas relações. Ela também permite proceder a análises mais acuradas de sensibilidade das soluções encontradas e obter estimativas para parâmetros não observáveis, por meio do procedimento de calibragem.

O texto está estruturado do seguinte modo. A seção 2 apresenta uma descrição sumária da abordagem sistêmica, visando a definir com mais precisão o conceito de "complexidade dinâmica", que, por ser uma característica intrínseca dos processos econômicos, aconselha a utilização dessa abordagem para estudar temas como o crescimento econômico. A seção 3 mostra como adaptar a essência do modelo de Solow-Swan a essa linguagem, e a seção 4 calibra a versão sistêmica do modelo para a economia brasileira no período 1970-2000 e testa sua validade. A seção 5 discute os resultados da simulação, inclusive a capacidade do modelo de prever a evolução das principais variáveis macroeconômicas brasileiras no período recente. A seção 6 conclui o trabalho, procurando destacar os benefícios de utilizar a metodologia de dinâmica de sistemas em estudos econômicos.

\section{2_A abordagem de dinâmica de sistemas}

Sistemas são conjuntos de elementos organizados intencionalmente pela ação humana ou que simplesmente se autoorganizam para cumprir propósitos específicos. O sistema elétrico de um automóvel é um exemplo do primeiro tipo, e uma biota não explorada por seres humanos, um exemplo do segundo tipo. Os sistemas econômicos são de um terceiro tipo que envolve tanto elementos de intencionalidade quanto de auto-organização. Os três tipos de sistema podem exibir graus consideráveis de complexidade, mas apenas os do segundo e do terceiro tipo definem-se como sistemas dinamicamente complexos. 
Complexidade dinâmica é uma propriedade que decorre do fato de que, em sistemas que a exibem, encontram-se presentes diferentes agentes que respondem a informações exógenas ou geradas pelo próprio comportamento do sistema. A presença dessa propriedade implica que o sistema pode responder de forma não intencional do ponto de vista dos agentes que tentam influir sobre ele, isto é, pressupondo que o sistema pode se auto-organizar. É fácil ver, então, que sistemas caracterizados por extrema complexidade de detalhes podem ser dinamicamente simples na medida em que não envolvam agentes capazes de responder a novas informações, e sistemas relativamente simples em termos de detalhes podem ser dinamicamente complexos.

A propriedade de auto-organização ou emergência ocorre porque, em sistemas dinamicamente complexos, as decisões individuais encontram-se interligadas em ciclos de retroalimentação (feedback). Sistemas dinamicamente simples evidentemente podem também envolver ciclos de retroalimentação, como no caso de um aparelho de ar-condicionado, que mantém uma temperatura pré-programada em um ambiente, mas, se devidamente projetados, não são capazes de gerar efeitos não pretendidos, salvo em caso de falha do equipamento. Já sistemas dinamicamente complexos, em que se encontram inseridos agentes, consequências não intencionais podem facilmente ocorrer, pelo simples fato de que, por exemplo, os agentes tentam antecipar, de forma muitas vezes difícil de prever, as reações dos demais.

A dinâmica de sistemas (system dynamics) é uma metodologia desenvolvida exatamente para rastrear os resultados de ações isoladas sobre o comportamento de variáveis que se encontram interligadas em malhas de retroalimentação, em que as relações entre causas e consequências estão geralmente distanciadas no tempo. Melhor dizendo, em que as variáveis relacionam-se com defasagens temporais normalmente não captadas em nosso modelo mental.

A metodologia de dinâmica de sistemas pode assim ser definida sinteticamente como abordagem informação/ ação/consequências, como representado na Figura 1.2

Novas informações levam a ações (fluxos) que alteram o estado (nível) das condições de um sistema após certa defasagem de tempo (as duas marcas paralelas sobre a seta indicam a existência de uma defasagem (delay) temporal entre a execução da ação e a mudança no es-
As técnicas incluídas nessa metodologia começaram a ser desenvolvidas pelo pesquisador do Massachusetts Institute of Technology Jay Forrester, na década de 1960, em uma série de estudos clássicos sobre economia regional e urbana, e vêm sendo empregadas em estudos aplicados em campos tão distintos do conhecimento, como política internacional, ecologia, gestão de recursos naturais e economia. Uma bibliografia de referência básica pode ser obtida em http:/ /www.systemdynamics. org/short_bibliography.htm. Uma referência básica para uma visão abrangente da metodologia e das principais técnicas é Sterman (2000).

2 Ver Coyle (1996). 
Figura 1_A abordagem de dinâmica de sistemas

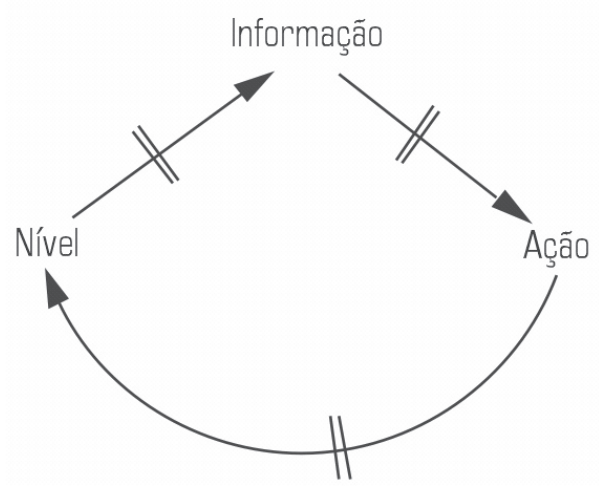

Fonte: Coyle (1996, p. 4)

tado do sistema). Ação, nível e informação interligam-se em dois tipos de ciclo de retroalimentação. $\mathrm{O}$ primeiro é o ciclo de realimentação negativa ou de equilíbrio em que o sistema reage a mudanças, compensando-as. O segundo tipo é o ciclo de realimentação positiva ou de autorreforço, em que o sistema amplifica eventuais perturbações. A próxima seção desenvolve um modelo para o processo de crescimento utilizando essa abordagem.

\section{3_Uma versão sistêmica do modelo Solow-Swan}

O estado de um sistema em um momento particular do tempo é dado pelo con- junto de variáveis de nível ou de estoque relevantes, as quais definem os fluxos que o sistema extrai ou devolve ao meio ambiente em que se localiza. O estoque de peixes em um reservatório, por exemplo, define o volume de pesca capaz de ser efetuado de modo a não levar o fluxo de nascimentos a cair abaixo do fluxo de capturas, o que levaria a população, e assim a capacidade de regeneração do sistema, a diminuir.

Os autores sistêmicos gostam de usar a analogia de enchimento de uma banheira para retratar a dinâmica dos sistemas estudados. Qualquer sistema será sustentável, de acordo com essa analogia, apenas se a vazão da torneira for maior ou igual à do ralo da banheira. Se isso não ocorrer, mesmo que por uma pequena margem, a banheira inevitavelmente se esvaziará. A condição de sustentabilidade ou de equilíbrio de um sistema pode ser assim definida intuitivamente em termos de equações em diferenças ou de equações diferenciais se utilizarmos um intervalo de integração suficientemente pequeno. O modelo de Solow-Swan, valendo-se de sua versão tradicional baseada em equações diferenciais, pode ser traduzido em uma versão sistêmica como mostrado na estrutura estoque-fluxo na Figura $2 .^{3}$ do modelo encontra-se no Apêndice. 
Figura 2_ 0 modelo de Solow-Swan em linguagem sistêmica

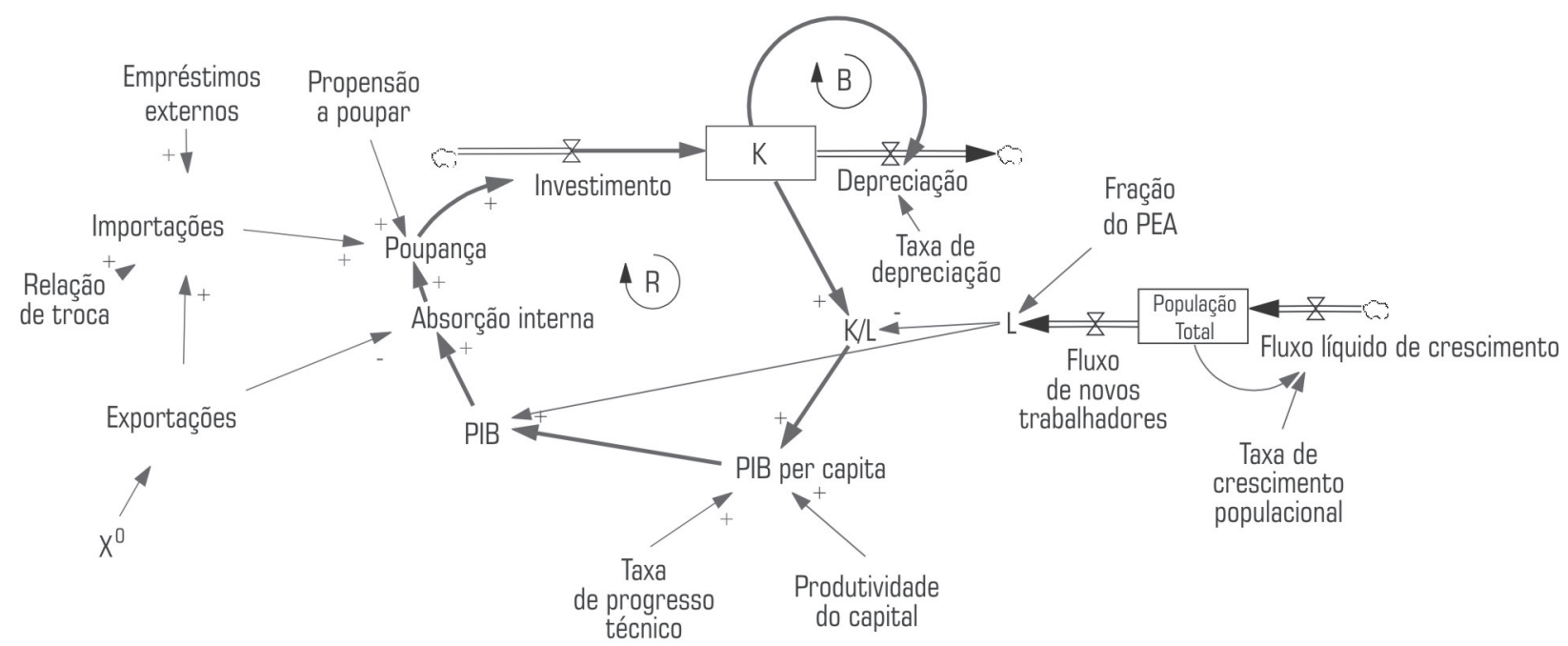

Fonte: Elaboração própria.

O estoque de capital de uma economia $(\mathrm{K})$, a principal das variáveis de nível do sistema, que são representadas no interior de retângulos, cresce com o fluxo de investimentos em bens de capital (no modelo de Solow não expandido) e decresce com o fluxo de depreciação, isto é:

$\mathrm{dK} / \mathrm{dt}=$ Investimento - Depreciação

Se esse estoque cresce mais rapidamente do que a população economicamente ativa (definida como uma fração da população total), a disponibilidade de capital per capita, K/L, aumenta, o que, dados a produtividade marginal do capital e o ritmo de progresso técnico, leva a uma maior produtividade, isto é, a um maior PIB per capita. O sinal positivo sobre o vínculo significa que existe uma relação direta entre as variáveis, e o sinal negativo, uma relação inversa. Assim, o crescimento populacional, tomado isoladamente, leva a uma redução na disponibilidade de capital per capita.

As exportações reduzem a absorção interna de bens, mas elevam a capacidade de importação e, assim, a dispo- 
nibilidade interna de oferta de bens de capital produzidos no exterior, elevando o nível de poupança bruta; quanto mais favorável for a relação de troca, isto é, a relação entre os preços internacionais dos bens domésticos e dos bens importados, maior o poder de compra internacional gerado por certo volume físico de exportações. O acesso a empréstimos externos que se somem à poupança interna, finalmente, permite em princípio aumentar as importações de bens de capital.

A condição de equilíbrio para o crescimento do estoque de capital é facilmente derivada da equação 1 , como:

Investimento $=$ Depreciação

A variável "Investimento" segue uma trajetória dada por um ciclo de realimentação positiva ou de autorreforço, assinalado em linhas mais grossas na figura com a letra $R$. O aumento do estoque de capital leva a uma maior dotação de capital por trabalhador. A maior dotação de capital permite, devido à produtividade do capital, crescimento do produto per capita. O crescimento do produto, por causa da propensão a poupar, viabiliza maiores níveis de poupança e, portanto, maiores níveis de investimentos em capital adicional.
Mas, além do ciclo de autorreforço R, existe outro ciclo, assinalado também em linhas mais grossas como $\mathrm{B}$, que tende a atenuar o crescimento, dado pela depreciação do capital: maiores níveis do estoque de capital levam a maiores fluxos de depreciação e, assim, a uma redução do estoque de capital. Observe-se que esse ciclo é de equilíbrio ou de estabilização, no sentido de que, se os investimentos forem inferiores à taxa de depreciação, o estoque de capital se reduzirá até o ponto em que o fluxo de capital se iguale à depreciação.

A interação entre esses dois ciclos reproduz a dinâmica básica do modelo Solow-Swan. A equação fundamental desse modelo (ver Barro e Sala-i-Martin, 2004, p. 30), em que, utilizando a clássica metáfora sistêmica já referida, a vazão da torneira é exatamente igual à do ralo da banheira, é análoga à condição de equilíbrio 2 :

$\dot{\kappa}=s \cdot f(k)-(n+\delta) \cdot k$

onde k é a dotação de capital por trabalhador, K/L, n é a taxa de crescimento populacional e $\delta$ é a taxa de depreciação média do estoque de capital. Isto é, a variação líquida do estoque de capital por trabalhador é igual à poupança per capita $=$ s.f(k), menos a soma da taxa de crescimento 
populacional e a depreciação (a lógica aqui é que o aumento do número de trabalhadores diminui o valor do estoque de capital por trabalhador da mesma forma que a depreciação).

Como a função de produção na forma intensiva $\mathrm{f}(\mathrm{k})$ em (3) está sujeita a rendimentos decrescentes no estoque de capital por trabalhador, enquanto o segundo termo cresce linearmente, é fácil concluir que haverá um ponto, definido como estado estacionário, em que o lado esquerdo da equação se anula e, portanto, a taxa de crescimento da dotação de capital por trabalhador torna-se igual a zero. O Gráfico 1 ilustra o processo.

A dotação de capital por trabalhador cresce a partir do nível $\mathrm{k}(0)$ porque nesse ponto a poupança e, portanto, o investimento bruto serão maiores do que a parcela do estoque de capital depreciada somada à parcela utilizada para empregar novos trabalhadores. O nível em que o estoque de capital se estabilizará (estado estacionário) será $\mathrm{k}^{*}$, no qual o investimento bruto será igual à depreciação. Desse modo, no modelo sem progresso técnico, a economia poderá cres-

\section{Gráfico 1_ 0 estado estacionário na acumulação de capital}

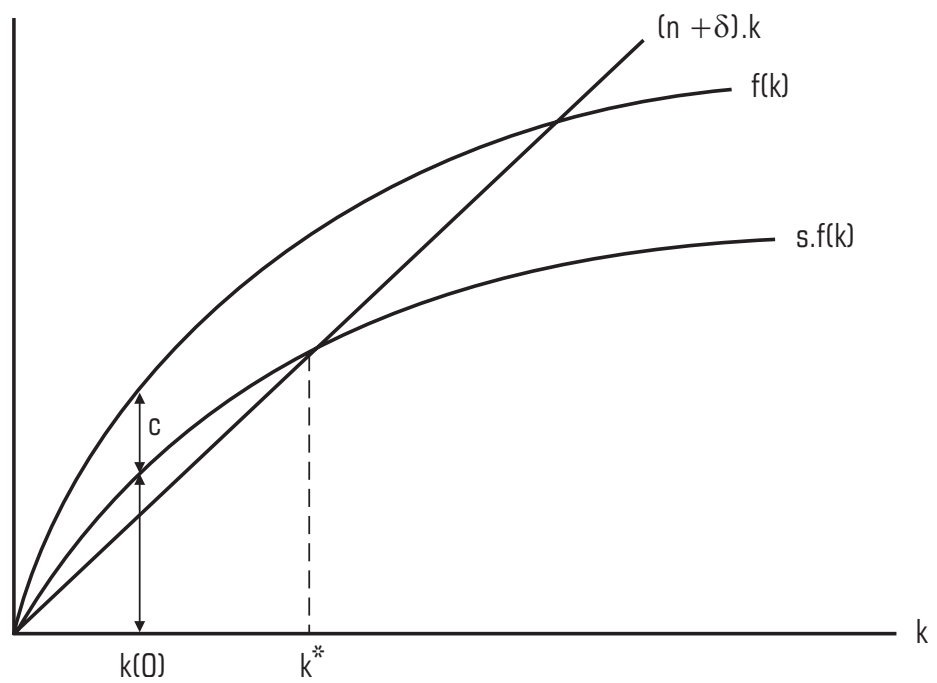

Fonte: Barro e Sala-i-Martin (2004, p. 29). 
4 Como a preocupação desse exercício é determinar o produto potencial da economia, assume-se que o País utilize uma parcela das importações, dada pela propensão a poupar, para complementar a oferta doméstica de bens de capital. Versões mais realistas do modelo deveriam utilizar uma especificação do tipo Mundell-Fleming, em que parte das importações é de bens intermediários e matériasprimas, e que, por isso, correlaciona-se positivamente com o nível de atividade. cer durante a transição de um nível de dotação de capital de equilíbrio para outro. Mas a única forma de uma economia apresentar uma taxa sustentável de crescimento ao longo do tempo é, então, deslocando progressivamente a função de produção $f(k)$ para cima, via introdução de progresso técnico, caso em que a equação 3 se transformaria em:

$\dot{k}=s . f[k, A(t)]-(n+\delta) \cdot k$

A única diferença com a equação (3), sendo que o nível de produto depende do nível de tecnologia A(t) (Barro e Salai-Martin, 2004, p. 54).

No modelo do Gráfico 1, o ciclo de autorreforço representa o primeiro termo da expressão para o investimento líquido, e o ciclo de equilíbrio, o segundo termo. O PIB per capita, f(k), e a poupança são dados, então, por:

$$
\begin{aligned}
& \mathrm{f}(\mathrm{k})=\mathrm{A}^{*}(\mathrm{~K} / \mathrm{L})^{\alpha} \\
& \mathrm{e} \\
& \mathrm{PIB}=\mathrm{f}(\mathrm{k})^{*} \mathrm{~L}
\end{aligned}
$$

onde A, $\alpha$ e L são, respectivamente, o nível de tecnologia, a produtividade marginal do capital e a população economicamente ativa.

A equação para a poupança total que, em equilíbrio, iguala os investimentos é dada por:
Poupança $=($ Absorção interna +Importações) * Propensão a poupar ${ }^{4}$

onde:

Absorção Interna $=$ PIB - Exportações

As relações externas são modeladas, simplificadamente, da seguinte forma:

Exportações $=\mathrm{X}^{0}$

Importações $=$ Exportações $*$ rela-

ções de troca + Emprestí-

mos internacionais

Relação de troca $=$ hrt + Índice médio de preços das exportações/Índice médio de

preços das importações

Empréstimos Internacionais $=\mathrm{E}^{0}$

Isso reflete as pressuposições simplificadoras de que as Exportações e os Empréstimos Externos são determinados exogenamente pela evolução do comércio internacional. As importações serão maiores para cada nível de exportações quanto mais favorável for a relação de troca e quanto maior o fluxo de empréstimos internacionais; hrt é um parâmetro de deslocamento dessa relação a ser estimado por calibragem.

O progresso técnico, finalmente, ocorre exogenamente, dado pela especificação a seguir, em que o valor de rpt se- 
rá calibrado nas simulações, como explicado na próxima seção:

$A=(1+\text { taxa de progresso técnico })^{t}$

Taxa de progresso técnico $=\mathrm{rpt}$

\section{Resultados}

As simulações para um período de 50 anos, a partir de 1970, a seguir, foram realizadas com o software Vensim (Ventana Systems); os parâmetros empregados correspondem às características da economia brasileira em 1970, tendo sido extraídos diretamente das séries históricas e de Bacha e Bonelli (2005), Bonelli (2005) e Morandi e Reis (2004); os valores monetários estão em bilhões de dólares de 1970. Os principais parâmetros do modelo de simulação encontram-se descritos no Quadro 1.

Nos Gráficos 2 a 7 apresentam-se algumas das simulações mais esclarecedoras do modelo, que confirmam as previsões do modelo Solow-Swan de que alterações de parâmetros da economia elevam o nível, mas não a taxa de crescimento de longo prazo do PIB.

Variações na propensão a poupar, como previsto pelo modelo de So-
low-Swan, produzem mudanças de nível na dotação de capital por trabalhador (K/L) e no PIB per capita; nesse sentido, aumenta a taxa de crescimento de transição - que ocorre quando a economia se desloca de um nível para outro -, mas não a taxa de crescimento de longo prazo, que é zero.

Variações nas relações de troca e na taxa de crescimento populacional têm o mesmo efeito. Um valor para a relação de troca de 0,5 significa que a razão entre os preços dos produtos exportados e dos importados é $50 \%$ maior do que o valor adotado como referência, 1 (a relação de troca é dada por $1+$ hrt, em que hrt é o parâmetro de deslocamento). O efeito apenas de nível da relação de troca sobre o PIB explica-se pelo fato de que, na especificação adotada neste trabalho, uma melhoria nessa relação aumenta a poupança bruta da economia e, assim, tem o mesmo efeito de um aumento de s.

Já o efeito da taxa de crescimento populacional corresponde ao aumento do produto devido ao aumento do emprego do fator trabalho, que, como o fator capital, está sujeito a rendimentos decrescentes, o que explica o efeito apenas de nível dessa variável. 
Quadro 1_Parâmetros do modelo de simulação

\begin{tabular}{|c|c|c|}
\hline Parâmetro & Valor & Descrição \\
\hline $\mathrm{D}$ & 0,05 & $\begin{array}{l}\text { Depreciação considerando vida útil média } \\
\text { dos equipamentos de } 20 \text { anos }\end{array}$ \\
\hline População & 93 milhões de pessoas & $\begin{array}{l}\text { Considerando uma taxa de crescimento populacional } \\
\text { de } 2,5 \% \text { a.a. até } 1990 \text { e de } 2,00 \% \text { a partir desse ano }\end{array}$ \\
\hline $\begin{array}{l}\text { Taxa de crescimento } \\
\text { populacional }\end{array}$ & $2,5 \%$ e $2,0 \%$ & Considerando uma queda na taxa a partir de 1980 \\
\hline PEA & 0,55 & A PEA é $55 \%$ da população total \\
\hline Estoque de capital inicial & US\$ 72 & Calculado com base na relação capital/produto de 1,8 \\
\hline Produtividade do capital & 0,35 & $\begin{array}{l}\text { Valor mínimo assumido nos estudos atuais; } \\
\text { tais valores variam entre } 0,35 \text { e } 0,5 \text {; sujeito a calibração }\end{array}$ \\
\hline Taxa de progresso técnico & 0,0075 & $\begin{array}{l}\text { Valor inicial para as simulações; } \\
\text { sujeito a calibração }\end{array}$ \\
\hline Absorção externa & $\begin{array}{l}\text { US\$ } \$, 5 \text { para o período } 1970-1985 \\
\quad \text { e US\$ } 11,5 \text { para } 1986-2000\end{array}$ & Valor mediano das exportações nos períodos \\
\hline Empréstimos & US\$ 5 & $\begin{array}{l}\text { Empréstimos para cada ano na segunda metade da } \\
\text { década de } 1970 \text {, em que o crescimento foi financiado } \\
\text { em grande parte por endividamento; o valor para os } \\
\text { demais anos é zero }\end{array}$ \\
\hline Relação de troca & 1 & Valor de referência; sujeito a calibração \\
\hline Propensão a poupar & 0,20 & $\begin{array}{l}\text { Valor usado convencionalmente nos estudos } \\
\text { aplicados; sujeito a calibração }\end{array}$ \\
\hline
\end{tabular}

Fonte: Elaboração própria. 
Gráficos 2 a 7 _ Efeitos de mudança nos parâmetros na dotação de capital por trabalhador e no nível do PIB "per capita"

K/L

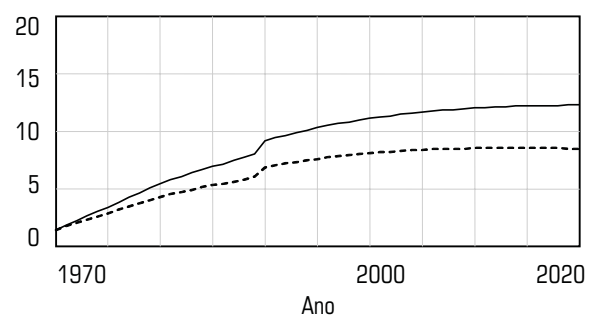

$\mathrm{s}=0,3$

$s=0,2 \quad \cdots$

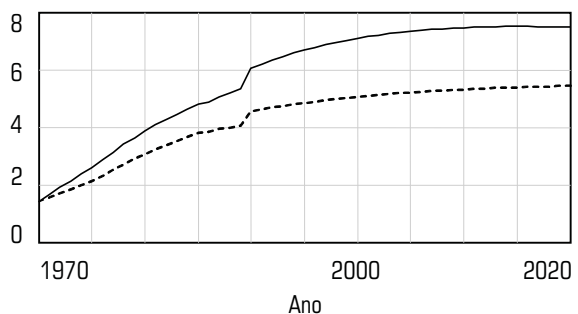

$\mathrm{rt}=0,5$

$r \mathrm{t}=-0,5$

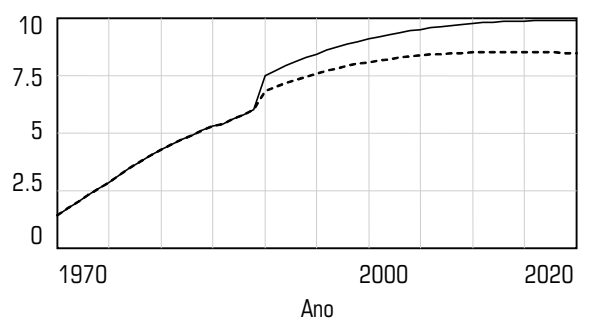

Taxa de crescimento populacional $=1,5 \%$

Taxa de crescimento populacional $=2 \%$

Fonte: Elaboração própria.

\section{PIB per capita}

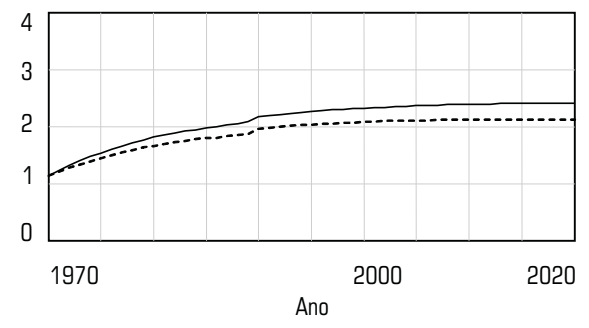

$\mathrm{s}=0,3$

$\mathrm{s}=0,2$

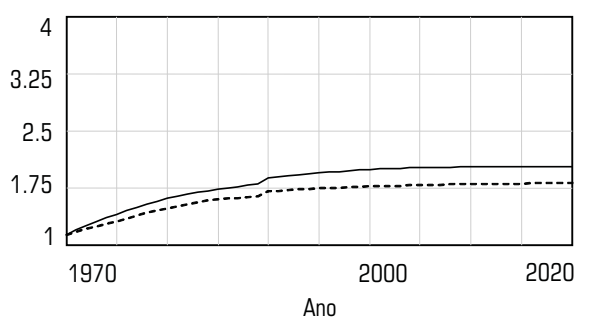

$\mathrm{rt}=0,5$

$r t=-0,5$

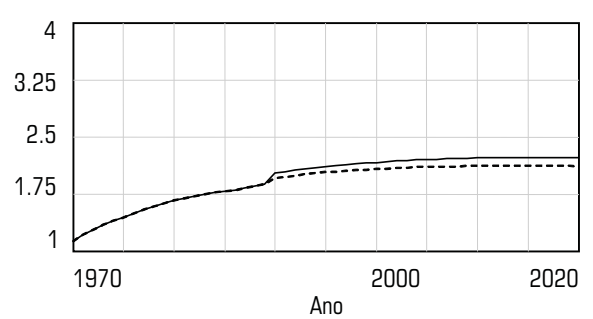

Taxa de crescimento populacional $=1,5 \%$ Taxa de crescimento populacional $=2 \%$ 
Mesmo investimentos em capital humano, que, no modelo de Solow aumentado pelo capital humano, teriam o efeito de aumentar a produtividade total do capital $(\alpha)^{5}$, teriam apenavs um efeito de nível, como mostrado nos Gráfi$\cos 8$ e 9 .

\section{Gráficos 8 e 9_ Efeito de investimentos em capital humano}

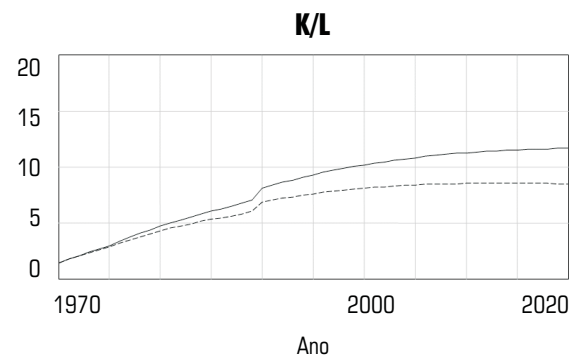

alfa $=0,5$

alfa $=0,35$

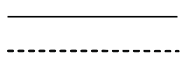

Ifa $=0,35$

Fonte: Elaboração própria.

Gráfico 10 e 11_Efeito de investimentos em infraestrutura geradora de progresso técnico

K/L

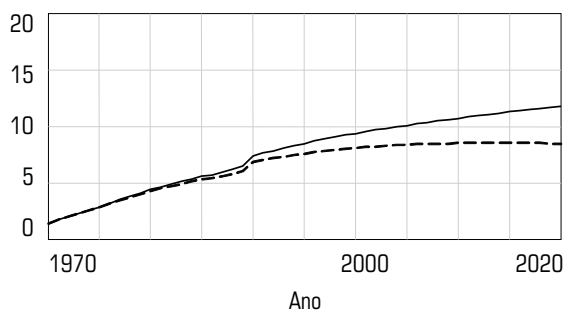

$\mathrm{rpt}=0,01$

$\mathrm{rpt}=0$

Fonte: Elaboração própria.
A única forma de elevar a taxa de crescimento de longo prazo seria, então, como fartamente sabido, gerar as condições para um processo sustentado de introdução de progresso técnico, como mostrado nos Gráficos 10 e $11 .^{6}$
5 Ver Barro e Sala-i-Martin (2004, p. 59-61).

6 A taxa de progresso técnico de 0,01 significa que o fator multiplicativo da função de produção (A) está crescendo a $1 \%$ ao ano.

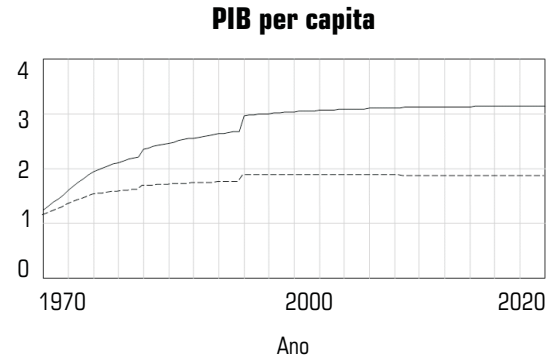

alfa $=0,5$
PIB per capita

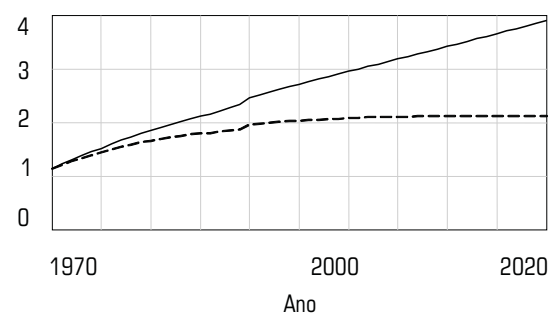

$r p t=0,01$

$\mathrm{rpt}=0$ 
Avaliar a pertinência de modelos sistêmicos - ou proceder à sua validação na linguagem do campo - é sempre um processo controverso, uma vez que as técnicas estatísticas tradicionais são de pouca valia em modelos de simulação. Há, entretanto, uma série de testes para estimar o grau de validade de um modelo sistêmico (ver Barlas, 1996).

Um primeiro teste é avaliar o grau em que o modelo é capaz de reproduzir a dinâmica real da economia que se está estudando. O Gráfico 12 indica que o grau de aderência do modelo, no sentido explicitado, é relativamente satisfatório, embora o modelo tenda a superestimar o valor do PIB no período final do experimento.

\section{Gráfico 12_Grau de aderência do modelo não calibrado}

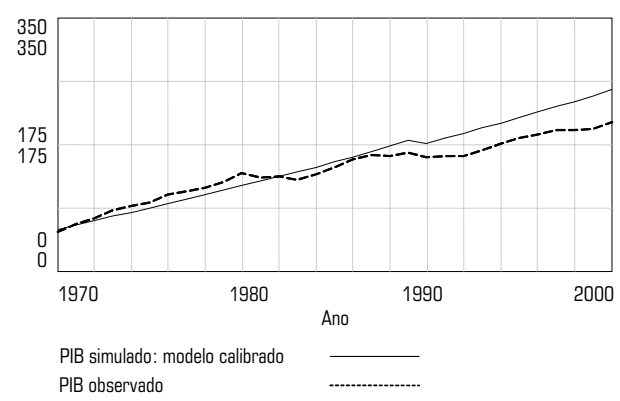

Fonte: Elaboração própria.
Para sanar essa deficiência, procedeu-se à calibragem do modelo como no Gráfico 13, a seguir, o que aumenta consideravelmente sua aderência à série de PIB real. Calibrar um modelo significa encontrar os valores das constantes que geram trajetórias das variáveis endógenas que melhor se ajustam aos dados reais correspondentes. Embora a calibração possa, em princípio, ser feita manualmente reduzindo os erros entre valores simulados e reais para cada variável, o Vensim permite calibrar automaticamente modelos complexos com grande número de variáveis endógenas e parâmetros.

\section{Gráfico 13_ Dinâmica do modelo calibrado}

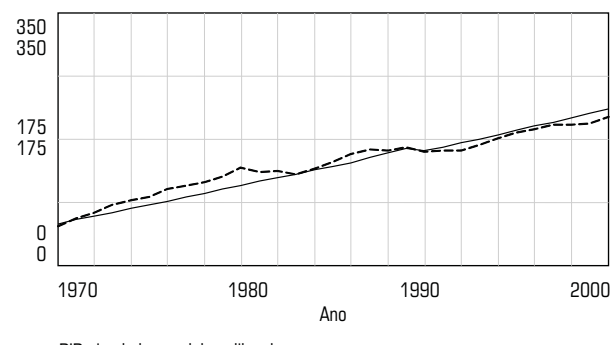

PIB simulado: modelo calibrado PIB observado

Modelo não calibrado: $\mathrm{s}=0,20 ;$ rtp $=0,0075 ; \alpha=0,35$; hrt $=1$ Modelo calibrado: $s=0,18 ;$ rtp $=0,005 ; \alpha=0,35$; hrt $=10$

Fonte: Elaboração própria. 
O procedimento é, em essência, o mesmo proposto por Kydland e Prescott (1982 e 1996).
Esse procedimento ${ }^{7}$ consiste em determinar a combinação de parâmetros que minimiza o erro entre valores simulados e observados das variáveis de controle do modelo sistêmico construído, isto é minimiza $\sum_{i=1}^{j} w_{i}\left(Y_{i}^{e}-Y_{i}^{r}\right)^{2}$, onde $\mathrm{j}$ e $\mathrm{w}_{\mathrm{i}}$ são, respectivamente, o número de variáveis a ser calibradas e a ponderação com que cada uma delas entra na função de "payoff", de modo que não seja dado peso maior no processo a variáveis com maior dimensão absoluta (para detalhes, ver Ventana Systems, 2005, cap. 18).

O presente modelo foi calibrado de modo a minimizar o erro (quadrado) entre as séries de valores estimados e observados do PIB, no período 1970 2000. Os parâmetros calibrados foram a propensão a poupar(s), a produtividade marginal do capital $(\alpha)$ a relação de trocas (rt) e a taxa de introdução de progresso técnico (rpt). A Figura 13 mostra que o modelo calibrado apresenta maior grau de aderência (visual) à série observada de PIB.

Se esses parâmetros tivessem de assumir valores patentemente irrealistas para que as simulações reproduzissem os dados observados da economia brasileira, não haveria razão para aceitar sua validade, mesmo que o modelo fosse eventualmente capaz de, dentro de cer- tos intervalos, gerar satisfatoriamente a dinâmica observada das variáveis de interesse. Mas não parece ser esse o caso.

Primeiro, a hipótese de propensão a poupar de 0,18 é consistente com a relação entre a Poupança Bruta e o PIB nos anos recentes. Textos atuais estimam a poupança bruta em torno de $20 \%$ do PIB por volta do ano 2000, após haver atingido um pico de $24 \%$ no início da década de 1980 (ver, por exemplo, Bacha e Bonelli, 2005; Castro e Hermann, 2005 e Apêndice Estatístico).

Segundo, o valor calibrado para o progresso técnico (rpt) de 0,5\% a.a. também não parece inconsistente com as estimativas obtidas em outros estudos. Silva Filho (2001) supõe o crescimento anual da PTF nos períodos 1980-1992 e 1993-2000, em -0,7\% e 0,9\%, respectivamente; Souza Júnior e Jayme Júnior (2004), em -2,0 e 0,5\%, e Bacha e Bonelli (2005), em - 0,8 e $1,2 \%$.

Terceiro, o valor da produtividade marginal do capital é consistente com outros estudos (Bacha e Bonelli, 2005), embora seja admissível que esse valor possa estar em alguma medida subestimado pelo procedimento de calibragem.

Por último, o valor do deslocamento da função de relação de troca (hrt) de 10 significa que o valor da rela- 
ção de troca, isto é, a razão entre o poder de compra das exportações sobre o preço das importações no que diz respeito ao ano-base de 1970 em 2000 é de 11, ou seja, onze vezes maior no período estudado do que no ano-base. As séries estatísticas disponíveis não refutam essa hipótese. A série de Capacidade de Importar do IPEA Data, por exemplo, indica um valor equivalente de hrt em 2000 de 4,1 e 9,9 em 2008 (os índices de capacidade para importar reportados na série histórica em 1970 e 2008 são de, respectivamente, 10 e 109).

Uma segunda forma de avaliar a pertinência desses modelos é testar sua robustez, no sentido especifico de que seus resultados básicos não sejam inaceitavelmente sensíveis a variações de parâmetros muitas vezes não diretamente observáveis, como a produtividade média do capital ou a taxa de introdução de progresso técnico (Gráfico 14 a 21).

\section{Gráficos 14 a 21_Análises de sensibilidade do modelo}

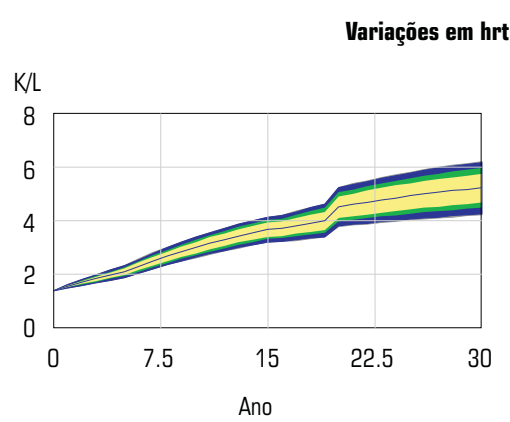

Variações em alfa $(0,35 \leqslant \alpha \leqslant 0,50)$
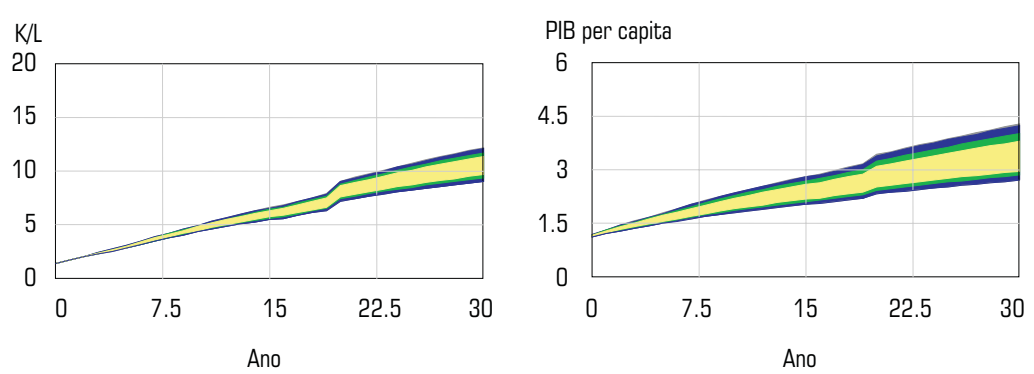


\section{Gráficos 14 a 21 _ Análises de sensibilidade do modelo}

\section{(conclusão)}

Variações na taxa de depreciação $(0,025 \leqslant d \leqslant 0,05)$

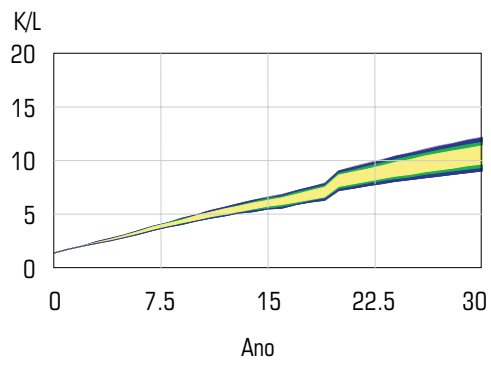

\section{PIB per capita}

Variações na taxa de progresso técnico $\quad(0,005 \leqslant r p t \leqslant 1,02)$
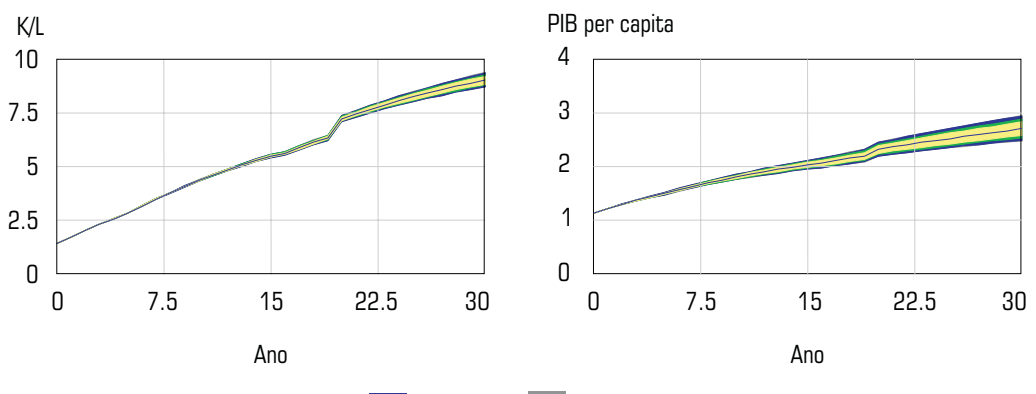

$50 \%$

$75 \%$

$95 \%$

$100 \%$

Fonte: Elaboração própria.

Notas: A ferramenta de análise de sensibilidade do Vensim simula automaticamente o modelo (por meio de métodos de simulação Monte Carlo) para variações aleatórias nos valores de parâmetros escolhidos dentro de intervalos preestabelecidos. Assim, por exemplo, define-se o intervalo de variação para a taxa de introdução de progresso técnico entre $0,5 \%$, o valor estimado por calibração neste trabalho para o parâmetro, e 1,2\%, a taxa estimada por Bacha e Bonelli (2005) para o período 1993-2002. Os dois últimos gráficos mostram que variar esse parâmetro não altera os resultados de forma significativa: as simulações para o PIB per capita e K/L distribuem-se em torno de U\$2.800 e 9 no último ano da simulação, respectivamente. Essa conclusão se mantém para os demais parâmetros. 
Uma última forma de "validar" um modelo sistêmico é testá-lo para condições extremas, por exemplo, supondo que a propensão a poupar ou a produtividade do capital sejam iguais a zero. Para passar no teste de validação, o modelo não pode apresentar trajetória inconsistente do ponto de vista lógico, mesmo nessas condições extremas. Diversos testes de dinâmica sob condições extremas foram efetuados, por exemplo, supondo s e $\alpha=0$, sendo que o modelo não apresentou dinâmicas inaceitáveis para as variáveis relevantes como PIB ou estoque de capital negativo.

\section{Discussão}

O Gráfico 22 apresenta as previsões do modelo de Solow-Swan em versão sistê- mica calibrada para a dinâmica do PIB brasileiro no período 2001-2007. Como o modelo foi calibrado exclusivamente para o período $1970-2000$, pode-se considerar este como tendo sido uma época de treinamento, e o período posterior, como o de previsão. A impressão óbvia é de que o modelo faz um trabalho muito bom em prever a evolução do PIB real nos últimos anos, o qual estaria convergindo para o nível de produto estimado com base no modelo de SolowSwan (Grafico 22).

As simulações de modo geral também confirmam os resultados encontrados por autores que recentemente têm procurado identificar as principais fontes do crescimento da economia brasileira, com diferenças pontuais:

\section{Gráfico 22_Evolução estimada do PIB: 2001-2007}

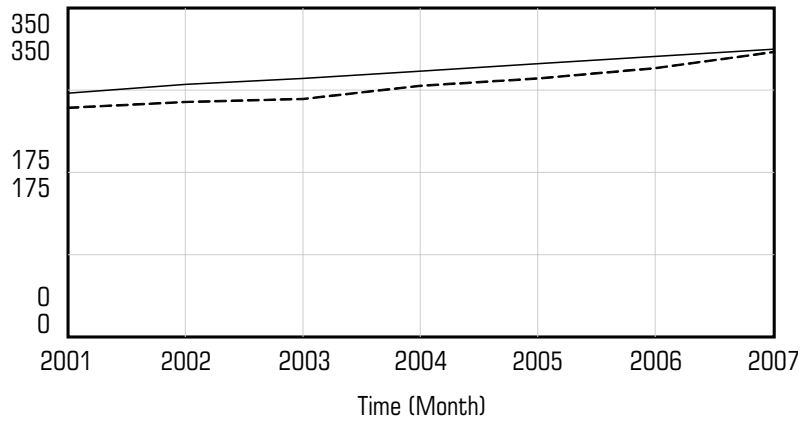

PIB simulado: Yr2007

PIB real: Yr2007

Fonte: Elaboração própria. 
8 Ponderando as taxas de crescimento da PTF por cada período estudado. Assim, por exemplo, a taxa de progresso técnico estimada para o período 1984-1993 é de -0,008, a qual entraria com uma ponderação de aproximadamente $33 \%$ no cômputo da taxa média do período.
- A taxa de progresso técnico tem sido em geral baixa; quando medida para todo o período, obtém-se a taxa de crescimento média de $0,5 \%$ ao ano; as taxas de progresso técnico da Coreia do Sul e de Taiwan para um período comparável, por exemplo, foram de $1,7 \%$ e $2,6 \%$ a.a., respectivamente (Barro e Sala-i-Martin, 2004, p. 440). Essa taxa, apesar disso, é ainda maior do que a estimada por Bacha e Bonelli (2005) para o período 1984-2002, que é de aproximadamente zero. ${ }^{8}$

- As relações de troca melhoraram substancialmente, refletindo principalmente a diversificação da pauta de exportações a partir de meados da década de 1980, como apontado pelo parâmetro hrt calibrado em um valor em torno de 10 .

- A taxa bruta de poupança, isto é, considerando a poupança doméstica e a interna, que melhor "ajusta" o modelo é de $18 \%$ para todo o período. Mas é possível identificar também que o impacto favorável da poupança tem sido declinante ao longo dos anos: os valores observados dessa variável em 1980 e 1975 , por exemplo, foram de $29 \%$ e $34 \%$, respectivamente.
- O impacto dos investimentos em capital humano sobre o crescimento do produto potencial tem sido insignificante, visto que a produtividade marginal do capital se situa no nível mais baixo entre todas as estimativas, $35 \%$.

- O crescimento do produto per capita no período pode ser em grande parte atribuído ao aumento da dotação de capital por trabalhador; enquanto a relação capital produto da economia elevou-se cerca de $2,9 \%$ a.a., a dotação de capital cresceu cerca de $5 \%$ a.a. em termos médios durante todo o período. As simulações, entretanto, revelam que esta taxa foi significativamente maior no início do período de simulação: entre 1990 e 2000, por exemplo, a taxa média da dotação de capital foi de apenas 1,9\% a.a., o que sugere que a economia esteja se aproximando do seu nível estacionário, como mostrado na Figura 2, e que, portanto, a contribuição desse fator para o crescimento tenda a diminuir de importância no futuro próximo.

- Parece provável também que não possamos esperar aumentos significativos na taxa de crescimento (de transição), provenientes de re- 
dução na taxa de crescimento populacional e em melhoria ulteriores das relações de troca. As análises de sensibilidade realizadas indicam que variação nesses parâmetros, além de produzir apenas variação de nível, não afeta significativamente a dinâmica de longo prazo da economia.

\section{6_Conclusão}

A conclusão geral a ser extraída do exercício de simulação realizado é relativamente pessimista. O Brasil ainda não foi capaz de alcançar uma trajetória de crescimento do progresso técnico consistente com uma taxa de crescimento aceitável. As fontes que até o momento sustentaram um crescimento relativo do PIB nas últimas décadas, como a intensificação da dotação de capital por homem, parecem estar atingindo seu teto de longo prazo. A possibilidade de elevar o nível do PIB per capita através de investimentos em infraestrutura e em educação, por outro lado, parece plausível, visto que a produtividade do capital, medida pelo parâmetro $\alpha$, encontra-se em níveis relativamente baixos e provavelmente pode aumentar para um valor de, digamos, 0,5, em consequência desses investimentos. Isso ocorrendo, o PIB per capita poderia aumentar em cerca de $50 \%$ quando o pleno impacto desses investimentos se fizesse sentir. Devese lembrar, entretanto, que esse seria um efeito de nível, isto é, não elevaria a taxa de crescimento de longo prazo em termos permanentes. Assim não parece haver muita saída para o País, como sugerido pela teoria e pela evidência empírica disponível, a não ser focalizar a política na constituição de um ambiente favorável à inovação tecnológica.

Mas em que medida as simulações realizadas podem ser tomadas como uma base confiável para fundamentar esta conclusão? Como todo modelo, o empregado neste trabalho é altamente irrealista, de modo que chega a ser surpreendente que ele seja capaz de gerar previsões tão consistentes com a evidência empírica disponível; nenhuma de suas previsões, como aferido pelos testes de condições extremas, é patentemente absurda ou mesmo desvia-se acintosamente dos dados observados. Mas será que faz realmente sentido fazer inferências com base em dados tão agregados?

Como é fácil suspeitar, achamos que sim e por uma razão simples. O crescimento de uma economia depende de 
Ver, por exemplo, Acemoglu et al. (2005) fatores estruturais os quais são em grande parte determinados por suas instituições. Como bem estabelecido na literatura mais recente, ${ }^{9}$ as instituições fundamentais de um país apresentam alto grau de estabilidade. Assim o ritmo de introdução de progresso técnico, por exemplo, depende, entre outras variáveis, do grau de estabilidade de instituições como o regime de proteção da propriedade intelectual e do grau de eficiência do Poder Judiciário em garantir a aplicação das leis correspondentes.

Por isso, embora seja plausível que esse ritmo possa diminuir em períodos de dificuldade econômica, faz sentido imaginar que, em uma perspectiva um pouco mais ampla, ele dependa de variáveis que se ajustam lentamente, o que torna convincente a ideia de que talvez seja mais realista utilizar uma mesma previsão dessa variável para períodos não excessivamente longos, mas também não tão curtos, como o estudado neste trabalho.

Dessa perspectiva, parece que exercícios como o que se realizou aqui podem ter mais um caráter esclarecedor do que investigativo, pois, afinal, as conclusões a que se chegou foram exatamente as previstas pela teoria econômica relevante e semelhantes às já obti- das em outros trabalhos. A contribuição mais importante que a metodologia da dinâmica de sistemas pode oferecer em estudos como este é a de, devido a sua flexibilidade, abrir uma gama de novas possibilidades no que diz respeito à experimentação com modelos teóricos, ao possibilitar, por exemplo, realizar análises de sensibilidade de soluções através de procedimentos simples e estimar o valor de parâmetros não observáveis com base no procedimento de calibração. A dinâmica de sistemas, em outras palavras, pode ser um instrumento eficiente para a escolha de políticas econômicas, particularmente as relacionadas com a questão do desenvolvimento econômico. 


\section{Referências bibliográficas}

ACEMOGLU, D.; JOHNSON, S.; ROBINSON, J. Institutions as a fundamental cause of longrun growth. In: AGHION, P.; DURLAUF, S. (Eds.). Handbook of economic growth. Vol. IA.

Elsevier, 2005.

BACHA, E.; BONELLI, R.

Uma interpretação das causas da desaceleração econômica do Brasil. Revista de Economia Politica, v. 25, n. 3, 2005.

BARLAS, Y. Formal aspects of model validity and validation in system dynamics. System Dynamics Review, v. 12, n. 3, 1996.

BARRO, R.; SALA-I-MARTIN, $\mathrm{X}$. Economic growth. Cambridge, Mass.: MIT Press, 2004.

BONELLI, R. O que causou o crescimento econômico no Brasil? In: CASTRO, L. B.; HERMAN, J. (Eds.). Economia brasileira contemporanea (1945-2004). Rio de Janeiro: Campus, 2005.

CASTRO, L. B.; HERMAN, J. (Eds.). Economia brasileira contemporânea (1945-2004). Rio de Janeiro: Campus, 2005.

COYLE, R. G. System dynamics modeling: a practical approach. London: Chapman and Hall, 1996.
DIEBOLD, F. The past, present and future of macroeconomic forecasting. Journal of Economic Perspectives, v. 12, n. 2, Spring 1998.

KREMER, M. Population growth and technological change: one million B.C. to 1990. The Quarterly Journal of Economics, v. 108, n. 3 , Aug. 1993.

KYDLAND, F.; PRESCOTT, E. The computational experiment: an econometric tool. Journal of Economic Perspectives, v. 10, n. 1, Winter 1996.

KYDLAND, F.; PRESCOTT, E. Time to build and aggregate fluctuations. Econometrica, v. 50, n. 6, 1982.

MORANDI, L.; REIS, E. J.

Estoque de capital fixo no Brasil: 1950-2002. In: ENCONTRO NACIONAL DE ECONOMIA, 32., 2004, João Pessoa.

Conferencicias... João Pessoa: ANPEC, 2004. p. 1-20.

SILVA FILHO, T. N. T. Estimando o produto potencial brasileiro: uma abordagem da função de produção. Brasília: Banco Central do Brasil, 2001.
SOUZA JÚNIOR, J. R.; JAYME JÚNIOR, F. Constrangimento ao crescimento no Brasil: um modelo de hiatos (1970-2000). Revista de Economia Contemporânea, v. 8, n. 1, 2004.

STERMAN, J. Business Dynamics. Boston: Irwin McGraw-Hill, 2000.

VENTANA Systems. User's guide. 2005. (Ventana Simulation Environment, DSS, Version 5, revision 2).

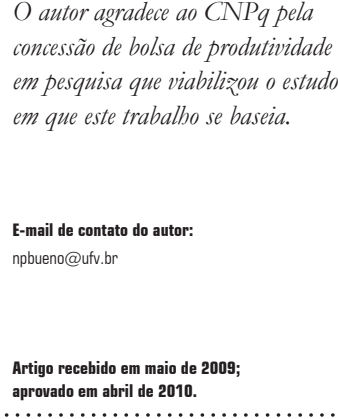


Apêndice

\section{Documentação Completa do Modelo de Simulação Calibrado}

$\mathrm{A}=(1+\text { taxa de progresso técnico })^{\wedge} \mathrm{t}$

Absorção Interna $=$ PIB - Exportações

alfa $=0,35$

$\mathrm{d}=0,05$

Depreciação $=\mathrm{K} * \mathrm{~d}$

$\mathrm{dK} / \mathrm{dt}=$ Poupança

$\mathrm{E}=5$

Empréstimos $=\operatorname{PULSE}(5,10) * \mathrm{E}$

Exportações $=$ " $\mathrm{x} *$ "

FINAL TIME $=30$

$\mathrm{rt}=1$

Importações $=$ Exportações $*$ relação de troca + Empréstimos

INITIAL TIME $=0$

$\mathrm{K}=$ INTEG (+ “dK/dt” - Depreciação, 72)

$\mathrm{L}=$ População $*$ PEA

$\mathrm{PEA}=0,55$

$\mathrm{PIB}=\mathrm{PIB}$ per capita $* \mathrm{~L}$

PIB per capita $=\mathrm{A} *(($ K/L”)^alfa $)$

População $=93 *(1+\text { taxa de crescimento populacional })^{\wedge} \mathrm{t}$

Poupança $=($ Absorção interna + Importações $) *$ Propensão a poupar

Propensão a poupar $=\mathrm{s}$

Relação de troca $=1+$ hrt

rpt $=0,005$

$\mathrm{s}=0,15$

taxa de crescimento populacional $=0,025-\operatorname{STEP}(0,005,20)$

taxa de progresso técnico $=\mathrm{rpt}$

TIME STEP $=1$

$\mathrm{x}^{*}=5,5+\operatorname{step}(16,6)$

PIBreal $=$ variável com dados reais 
